\title{
Estado de la cuestión sobre la perspectiva poscolonial: Subversión de conceptos en literatura infantil y juvenil actual
}

\author{
Maria Luisa Alonso Soroa \\ Universitat Autònoma de Barcelona, Barcelona, España
}

(Artículo recibido el 30 de noviembre de 2011; versión final recibida el 30 de enero de 2012)

\begin{abstract}
Partiendo del análisis de las obras recientes más emblemáticas sobre la teoría poscolonial y de los trabajos críticos existentes sobre su aplicación en el campo de la literatura infantil y juvenil, este artículo pretende, por un lado, llamar la atención sobre la necesidad de extender la aplicación de la teoría poscolonial en el estudio de este tipo de literatura para redefinir una idea desvirtuada de multiculturalismo y, por otro lado, reconocer la utilidad de este enfoque a la hora de examinar las construcciones de la imagen del mundo en que vivimos que se hacen en textos de literatura infantil y juvenil actual en países occidentales. El artículo introduce en qué consiste la perspectiva crítica poscolonial y analiza su relación con los movimientos migratorios y con la condición híbrida de la cultura para así explicar el interés de aplicar esta perspectiva en el campo de estudio de la literatura infantil y juvenil actual. Se concluye ofreciendo el ejemplo de un texto poético para jóvenes lectores que ilustra cómo su autora rompe con el antiguo discurso imperialista entre colonizador y colonizado y muestra una imagen poco estereotipada de la infancia.
\end{abstract}

\section{Sobre la complejidad del poscolonialismo}

"La poscolonialidad, por su parte, es un saludable recordatorio de las persistentes relaciones neocoloniales dentro del nuevo orden mundial y la división multinacional del trabajo” (Bhabha 1993:9)

Los estudios acerca de lo poscolonial han suscitado en las últimas décadas un interés creciente y su popularidad y diversificación han transcendido el campo de lo literario, ámbito de estudio donde se fraguó la teoría poscolonial. Si bien la obra de los más prestigiosos -y veteranosteóricos del discurso colonial como Edward Said, Gayatri Spivak y Homi Bhabha “continúa

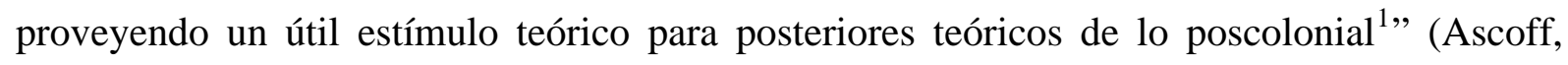
Griffiths, Tiffin 2006, cap. 6, sec. 1 § 2), la expansión del poscolonialismo y su polarización han dado lugar a intrincadas discusiones acerca del acotamiento de este campo de estudio, empezando por la propia definición del qué y quién debe ser considerado poscolonial. Sin entrar en detalle acerca de los distintos elementos que suscitan polémica y los diversos 
enfoques teóricos que han ido surgiendo, pues son discusiones que permanecen abiertas, sí es necesario escoger una definición de lo poscolonial que poder usar como punto de partida y encuadramiento de este artículo. Parece apropiado definir el término poscolonial en un sentido amplio como "toda la cultura afectada por el proceso del imperialismo desde el momento de la colonización hasta hoy en día” (Ascoff, Griffiths, Tiffin 2006, Intro. sec.1 § 2) y de forma más precisa considerarlo como "un término para las diversas y a menudo contestatarias formulaciones de la producción cultural de gentes colonizadas más que como una disciplina o metodología per se” (Ascoff, Griffiths, Tiffin, 2006, cap. 6, sec. $1 \S 2$ ). Puesto que el lenguaje es sin duda una importante herramienta para desarrollar estrategias de descolonización, en el campo de la literatura el enfoque pertinente es utilizar "la potente crítica sistemática de la gran narrativa eurocéntrica que constituye el poscolonialismo, como mero punto de partida”, tal y como sugiere Shaboo Xie en el prefacio de la obra sobre poscolonialismo en literatura infantil Voices of the other (McGillis, 2000: 1).

La validez de los estudios poscoloniales debe residir, por tanto, en su eficacia para comprender y poder actuar en el mundo que nos rodea. Para realizar de forma efectiva cualquier análisis de narrativas actuales relacionadas con el poscolonialismo, es necesario entonces recalcar su íntima conexión con el neocolonialismo ya que, como citaba Jan Mohamed, el mundo "en vez de haberse emancipado completamente a sí mismo del imperialismo, permanece atrapado en el neocolonialismo o la fase hegemónica del imperialismo" (citado en Jauregui, Moraña y Mignolo, 2008: 2).

\section{Poscolonialismo y las voces de la diáspora}

Los movimientos de poblaciones más importantes que ha registrado la humanidad han sido el resultado deliberado del imperialismo. Las inequidades actuales entre norte y sur han tenido y siguen teniendo como resultado importantes movimientos migratorios. La llegada de poblaciones considerables procedentes de lo que un día fueron colonias al corazón de lo que antiguamente era el imperio, “crea condiciones bajo las cuales estos grupos pueden en algún sentido ser considerados como poscoloniales” (Edwards 2008: 13). Al igual que los poderes coloniales interfirieron en su día numerosas culturas indígenas de entonces, como Bhabha sugiere, "los grupos migratorios poscoloniales podrían ser vistos como que devuelven el favor por modesta que sea la forma” (1993: 13).

Siguiendo la perspectiva ideológica de Edward Said sobre el periodo colonial según la cual el Otro colonizado es una construcción occidental, una proyección de los miedos y 
deseos ocultos tras una concepción monolítica del mundo de corte eurocentrista; la idea básica de la que se parte aquí es que los grupos poscoloniales están lejos de ser ajenos o el Otro para las identidades occidentales y que esta idea adquiere mayor veracidad en el periodo poscolonial cuando es el Otro el que viene a Occidente. La utilidad del poscolonialismo con relación a este fenómeno de los desplazamientos humanos no reside en ofrecer una visión del globalizado mundo actual en términos de bipolaridades (colonizador-colonizado, centroperiferia, opresor-oprimido) sino más bien en el enorme poder de invención creativa del “espacio de intervención que emerge en los intersticios culturales” (Bhabha, 1994:51) y en su capacidad de "interrogar y subvertir las formaciones culturales derivadas del imperialismo" (Ascoff, Griffiths, Tiffin, 2006, Intro. sec.6 § 5), que yace precisamente en su carácter híbrido. Esta hibridez cultural no debe ser vista como una propiedad de culturas minoritarias que conviven de forma paralela a identidades culturales nacionales soberanas y ni siquiera se debe comprender como un rasgo secundario o tal vez exótico de las sociedades de nuestros tiempos, sino que precisamente la hibridez cultural es su principal característica.

Como se puede observar analizando la función social de textos literarios durante los periodos coloniales, “la literatura ofrece una de las vías más importantes a través de las cuales (..) las realidades diarias de los colonizados han sido más profundamente reveladas y más profundamente influyentes” (Ascoff, Griffiths, Tiffin 2006, Intro. § 1). Mediante distintas formas de expresión literaria, voces procedentes de grupos poscoloniales reflejan hoy en día formas de comprender el mundo no-eurocentristas y hacen eco en sus narrativas de su rechazo del lenguaje como "medio a través del cual se perpetua una estructura jerarquizada de poder y se establecen concepciones de verdad, orden y realidad” (Ascoff, Griffiths, Tiffin, 2006, Intro. sec. $5 \S 1)$.

\section{Literatura infantil y juvenil y poscolonialismo}

Para examinar la relación entre literatura infantil y poscolonialismo es necesario analizar en primer lugar la idea de multiculturalismo para comprender que, mientras éste último fue un concepto surgido de forma pragmática para tratar de definir un contexto cultural de difícil definición, y cuya expansión en el tiempo y espacio se ha hecho de forma difusa comprometiendo su eficacia; los estudios poscoloniales son el fruto de un reflexivo y comprometido trabajo de pensadores implicados en la construcción de una perspectiva crítica profunda. 
Multiculturalismo, al igual que feminismo, fueron “asuntos calientes” en gran parte de los países desarrollados en las décadas de los 70 y 80, y la necesidad de ofrecer a niños y jóvenes una literatura que colaborara con una educación en la diversidad cultural es una idea globalmente reconocida desde entonces. Las políticas de educación de países receptores de grupos migratorios contribuyeron en gran medida a difundir el multiculturalismo. En el terreno de la crítica, desde los años 70 proliferaron en el campo de la literatura infantil y juvenil "los estudios sobre la imagen sociocultural que se desea transmitir a los niños y niñas (y que describen) corpus literarios desde preocupaciones ideológicas como la convivencia multicultural” (Colomer, 2003:106).

Si bien el multiculturalismo fue una idea revolucionaria ${ }^{2}$ cuya presencia en el paisaje cultural “tuvo gran influencia en la representación que cada uno se hacía de sí mismo y de sus relaciones con los demás” (Paul, 2005: 231), la necesidad de insertar a cualquier precio lo multicultural en políticas y agendas educativas provocó la pérdida de su sentido radical impulsor de los cambios sociales. Esto último trajo como consecuencia, entre otras, la expansión y predominio de un multiculturalismo de tipo "boutique”, término acuñado por Fisch en su obra Boutique Multiculturalism, or Why Liberals Are Incapable of Thinking about Hate Speech, que describe el tipo de libros infantiles que dominaban la escena a finales del siglo XX, “caracterizado por sus relaciones superficiales y cosméticas con los objetos a los que afecta” (citado en Paul, 2005:232). Si tenemos en cuenta que hasta la década de los 80 (y en muchos lugares más tarde), "la mera presencia de un niño de color era suficiente para clasificar un libro de multicultural y que éste era en general el tipo de libros que entraba en escuelas y bibliotecas” (ibíd.), junto con el hecho de que muchos de los libros etiquetados como multiculturales eran, y algunos siguen siendo, de corte paternalista del tipo en que el narrador asume de alguna manera que chicos extranjeros llegados a sociedades eurocentristas envidian la vida de esas sociedades y anhelan sus formas de vida; es fácil llegar a la conclusión de que el multiculturalismo como elemento de juicio en el ámbito de la literatura infantil y juvenil debe ser reconsiderado. No se debe olvidar que la infancia, y la literatura para la infancia por extensión, es un momento o lugar “clave para desarrollar el potencial individual necesario para construir un futuro en el que las que las sociedades pudieran llegar a ser diferentes en distintas formas relevantes” (Reynolds, 2007:100).

Esta pérdida de sentido radical que Lisa Paul ha llamado "los usos y abusos del multiculturalismo” (2005: 232), lleva apareada la pérdida de la eficacia del término, pues su banalización merma la fuerza de cualquier discurso efectivo sobre diversidad cultural, 
obstaculizando la función de la literatura infantil y juvenil de transformación social y estética de la cultura que requiere "motivar a los lectores a abordar ideas, asuntos y objetos desde nuevas perspectivas y así preparar el camino al cambio” (Reynolds, 2007:99). El desgaste del multiculturalismo nos alerta sobre el hecho de que "no se deben subestimar las dificultades de un pensamiento internacional e intercultural” (Hunt, 2003:26) y de la necesidad de crear nuevas perspectivas críticas en el campo de estudio de la literatura infantil y juvenil. Se hace necesario, pues, acercarnos a la literatura infantil y juvenil adoptando un enfoque serio y profundo como el poscolonial que redefina el multiculturalismo ${ }^{3}$ y que aporte a sus lectores la visión necesariamente sincrética e híbrida del mundo en que vivimos. Puesto que el multiculturalismo de tipo boutique sigue siendo el enfoque dominante que encontramos en la literatura infantil y juvenil actual, resulta de gran utilidad una perspectiva crítica que aborde de forma sistemática asuntos tales como en qué medida en los libros para niños y jóvenes actuales

el neocolonialismo se manifiesta a sí mismo tanto como una descripción/definición de culturas minoritarias como empedernidamente otras e inferiores de alguna manera con respecto a la cultura europea o eurocéntrica, o como una apropiación de otras culturases decir una asimilación de culturas minoritarias en la corriente dominante de pensamiento. (McGillis, 2000:24)

El enfoque poscolonial de la literatura no es universalista sino que trata fundamentalmente de huir de la idea de que un centro impone su criterio como universal. Se trata de un enfoque que no impone ni valores ni significados como características intrínsecas de los textos, sino que considera cómo se inscriben en ellos "las relaciones cambiantes de poder y conocimiento evidentes en las sociedades coloniales y poscoloniales y en sus discusiones de narrativas tradicionales los estudios literarios poscoloniales resisten interpretaciones universalizantes” (Bradford 2007: 8). La fuerza de la teoría poscolonial se revigoriza en los textos para jóvenes lectores que abordan cuestiones de resistencia, poder, etnicidad, nacionalidad, lenguaje y cultura. La transformación en este tipo de literatura de los discursos dominantes "provee (a sus jóvenes lectores) modelos importantes para comprender el lugar de lo local en un mundo crecientemente globalizado” (Ascoff, Griffiths, Tiffin, 2006, Cap.6 , § 5).

$\mathrm{Si}$, como se acaba de mencionar, las relaciones cambiantes de poder son características del mundo actual, lógicamente reflejadas en la literatura infantil y juvenil, que son el punto de mira de la perspectiva poscolonial; es fácil comprender que la visión predominante de este enfoque crítico al abordar estos textos no es que ellos sirvan para "asegurar al niño en la cultura” (Reynolds, 2007: 103), pues adscribiría a la literatura infantil "una visión 
coercitivamente normalizadora” (Reynolds, 2007: 108) y poco esperanzadora, sino que pretende reclamar que el potencial de la literatura para los más jóvenes reside precisamente en su fuerza transformadora.

Esta capacidad transformadora, de agente social, es el compromiso intelectual que reclaman precisamente Said, Bhabha o Spivak con sus aportaciones a la teoría poscolonial. De este modo, uniendo un potencial y otro de transformación social, se deduce que las estrategias subversivas en textos poscoloniales para jóvenes lectores entrañan una capacidad de compromiso político y social especial.

Sin embargo, a pesar del potencial señalado, el análisis poscolonial de textos infantiles es relativamente reciente, pues, como explica Claire Bradford, “desde la publicación en 1978 del libro de Edward Said Orientalismo, tomada como el inicio de los estudios poscoloniales, se necesitó más de una década para que el mundo académico de la literatura infantil comenzara a implicarse con la teoría poscolonial y su potencial crítico” (2007: 7) y su aplicación prácticamente se reduce al ámbito de la literatura escrita en inglés o al abordaje de textos producidos por quienes un día fueron gentes colonizadas en antiguas colonias. Pocos son los libros y escasos los artículos académicos en el ámbito de la literatura infantil -y aún más escasos en las letras hispanas ${ }^{4}$ - que aborden textos actuales bajo la perspectiva poscolonial.

Bradford, al tratar de buscar una explicación al aparente desinterés de los estudios poscoloniales por la literatura infantil y juvenil, acude precisamente a la estrecha vinculación de la teoría poscolonial con neocolonialismo y al predominio en la literatura infantil de posiciones universalizantes homogeneizadoras.

una de las razones [de este descuido] yace en el aún prevaleciente dominio, tanto en los libros infantiles como en el debate crítico sobre estos, de las formas de pensamiento humanístico liberales, que enfatizan lo que los humanos tienen en común a lo largo del tiempo y del espacio y dejan poco lugar para lecturas más historicistas y politizadas. (2007: 9)

Un trabajo interesante con relación a la complejidad de las construcciones del mundo reveladas en textos infantiles poscoloniales es el del australiano Graheme Harper, centrado de forma especial en ficción anglosajona contemporánea: As if by magic: World creation in poscolonial children's literature. En este estudio, su autor adopta un enfoque metodológico que podría ser tenido en cuenta a la hora de sintetizar otros corpus literarios bajo una perspectiva poscolonial (Ej.: ficción infantil contemporánea escrita en español en las dos últimas décadas). Según Harper, el enfoque de Goodman que él utiliza y que se caracteriza 
por cinco procesos (composición y descomposición, peso, orden, supresión y complementariedad y deformación) “es un método heurístico ejemplar para abordar ficción contemporánea infantil” (1997: 42). Apoyado en el citado enfoque metodológico, Harper aborda con suficiente profundidad importantes cuestiones como la condición híbrida del lenguaje, el diálogo entre comunidades que llegan y que se van, el reconocimiento mutuo de la deslocalización cultural, las asimetrías en las relaciones de poder o el hecho de que "la hibridación cultural no conduce a la falta de autenticidad” (1997: 47).

La académica Lisa Paul, citada en párrafos anteriores, es otra prestigiosa autora de crítica sobre literatura infantil y juvenil que ha prestado atención a textos poscoloniales en los que es "el imperio el que escribe su respuesta”. Paul argumenta que los textos que nos traen de forma viva a la literatura infantil voces pertenecientes a grupos poscoloniales, resultan especialmente atractivos para los niños porque sienten una especial empatía hacia las expresiones de gentes colonizadas, pues son ellos las últimas gentes colonizadas que quedan.

Lisa Paul hace referencia con sus argumentos a la conocida perspectiva del también canadiense Perry Nodelman (1992), que establece un paralelismo entre la teoría de Said y la literatura infantil, entre subalternidad ${ }^{6}$ e infancia. Según esta perspectiva, la infancia constituye un grupo colonizado sobre el cual escriben los adultos de la misma manera que quien hablaba por los orientales eran los orientalistas. Este punto de vista, si bien tiene sus detractores -como Bradford, que no considera apropiado el paralelismo mencionado- sostiene que la visión poscolonial de la literatura infantil y juvenil es extensible a todo este tipo de literatura y no sólo a los textos para jóvenes lectores que abordan directa o indirectamente el desmantelamiento de conceptos poscoloniales.

Por su parte, Peter Hunt parafraseando a Nodelman en el VII Simposio internacional de la Sociedad Española de Didáctica de la Lengua y Literatura, decía que "la literatura infantil ha sido en efecto una literatura colonizada” (2003:25) y parafraseaba en pasado porque este autor reivindica que en el panorama actual los importantes cambios que se están produciendo en las estructuras de poder dentro del mundo de la educación deberían revolucionar el campo de la literatura infantil y juvenil, pues crean la oportunidad y necesidad de un nuevo criticismo: "solo habrá una no-colonización, una no-continuación de la lucha de poder si el criticismo en el área de la literatura infantil se limita a ser una intervención”(ibíd.). Como este autor es un defensor de la naturaleza única y especial de la literatura infantil, el modelo de criticismo por el que aboga es necesariamente diferente del criticismo aplicado a la literatura general que dice debe ser un modelo de “cooperación en vez de confrontación, de 
síntesis y no análisis, de lecturas e interacciones individuales, no de verdades universales” (ibíd.). Según Hunt, uno de los potenciales de cambio del criticismo en el terreno la literatura infantil es el desarrollo del poscolonialismo. Siguiendo esta postura, es necesario, al igual que se ha hecho con la literatura poscolonial femenina ${ }^{7}$, tener en cuenta la especificidad de la literatura infantil a la hora de examinarla bajo un enfoque poscolonial.

\section{Algunas voces poscoloniales para jóvenes lectores}

Parece apropiado concluir este artículo examinando un ejemplo de voces poscoloniales que tratan de desmantelar conceptos heredados del colonialismo y perpetuados por el orden establecido, como el que constituye la poesía escrita para niños y jóvenes por un grupo de poetas caribeños activos en el Reino Unido que, empezando con James Berry en los años 70, ha ido incorporando a poetas como Grace Nichols, Valerie Bloom, Benjamin Zephaniah o John Agard. Este grupo de poetas afrocaribeños puede ser considerado parte del segundo de los dos grandes movimientos que según Peter Hunt forman la poesía infantil británica desde los años 70, “uno de ellos dirigido a llevar temas serios a formas más o menos tradicionales y el otro orientado hacia una poesía ligera, divertida y simple derivada del verso popular y los juegos infantiles” (2001: 296).

Estos poetas introducen en la poesía infantil ritmos diferentes, revitalizan el lenguaje y abordan, desde nuevas perspectivas, construcciones no universalistas del mundo y de la infancia. Sus poemas no representan la "versión idealizada de multiculturalismo” (Paul 2005: 233) de la corriente dominante, sino que se comprometen subvirtiendo la demoledora máquina de la cultura masiva. Este tipo de poesía, de escasa identificación y reconocimiento en Europa, es una respuesta nacida de la sensibilidad cultural del tiempo en que viven estos poetas y es un "desarrollo inevitable en el contexto actual de evolución del mundo y de la multiculturalidad” (Hunt, 2001: 298).

El siguiente poema, Wha Me Mudder Do (en 100 Best Poems for Children, Puffin), escrito por la poetisa guyanesa Grace Nichols en 1988, brinda la oportunidad de examinar cómo estos poetas hacen uso de ritmo y lenguaje para mostrar construcciones poco convencionales de la infancia y del mundo en que vivimos. 


\section{Wha Me Mudder Do}

Mek me tell you wha me mudder do wha me mudder do wha me mudder do

Me mudder pound plaintain mek fufu Me mudder catch crab mek calaloo stew

Mek me tell you wha me mudder do wha me mudder do wha me mudder do

$$
\begin{aligned}
& \text { Me mudder beat hammer } \\
& \text { Me mudder turn screw } \\
& \text { she paint chair red } \\
& \text { then she paint it blue }
\end{aligned}
$$

Mek me tell you wha me mudder do wha me mudder do wha me mudder do

$$
\begin{gathered}
\text { Me mudder chase bad-cow } \\
\text { with one 'Shoo' } \\
\text { she paddle down river } \\
\text { in she own canoe } \\
\text { Ain't have nothing } \\
\text { dat me mudder can't do } \\
\text { Ain't have nothing } \\
\text { dat me mudder can't do } \\
\text { Mek me tell you }
\end{gathered}
$$

Siguiendo con la célebre afirmación anteriormente mencionada de Rushdie, en este poema se distingue la voz del colonizado que ahora contesta al colonizador, es decir, Grace Nichols ofrece aquí una visión alternativa del tan nombrado encuentro colonial. La intención de la voz infantil del poema, tan segura de sí misma, es subrayar la necesidad de que el lector sea consciente de todo lo que su madre es capaz, de hacer comprender al lector que la infancia puede ser diferente de cómo un niño o niña occidental normalmente la imagina o vive, que también puede ser como la que se describe en el poema, una infancia muy apetecible vivida al otro lado del océano en estrecho contacto con la naturaleza, con madres valientes, atractivas, dinámicas y amorosas que aunque cocinan cosas raras son capaces de hacer de todo.

Las características de este atractivo y rítmico poema de Grace Nichols parecen propiciar la capacidad de permanecer en la memoria del niño y de continuar resonando en el 
futuro y se puede decir que la forma que el poema tiene de representar el mundo (la maternidad, la infancia, la cultura) supone un buen ejemplo de contribución literaria para la construcción de una conciencia verdaderamente intercultural y de un mundo más tolerante.

\section{Conclusión}

Este artículo ha pretendido contextualizar la pertinencia de extender una mirada poscolonial de la literatura para los más jóvenes, poniendo en relieve la reciente y aún escasa atención de los estudios poscoloniales en este campo. Consciente de que la literatura infantil y juvenil es un importante foro para la transmisión y cuestionamiento de ideologías dominantes y construcciones sociales como las heredadas del colonialismo e imperialismo, la autora quiere contribuir a una educación en la tolerancia llamando la atención en distintos escenarios relacionados con la educación, la infancia y la juventud, sobre la necesidad de dedicar recursos a la identificación y reconocimiento de voces vivas contemporáneas de grupos poscoloniales que reflejen en la literatura infantil la naturaleza necesariamente híbrida de la cultura actual.

\section{Referencias bibliográficas}

Ascoff B., Griffiths G., Tiffin H. (1989/2006). The empire writes back: theory and practice in postcolonial literatures. Nueva York y Londres: Routledge. Kindle e-reader version de www.amazon.com

Bradford, C. (2007). Unsettling narratives: postcolonial readings in children's literature. Toronto: Wilfrid Laurier.

Bhahba H. (1994). The location of culture. Nueva York y Londres: Routledge classics.

Colomer T. (2003). La formación del lector literario. Salamanca: Fundación Germán Ruipérez.

Edwards J. (2008). Postcolonial literature. Basingstoke: Palgrave Macmillan.

Harper G. (1997). As if by magic: world creation in Postcolonial Children's literature. A Review of International English Literature, 28(1): 1-14.

Hunt, P (2001). Children's literature. Oxford: Blackwell Guides for Literature.

Hunt, P (2003). Exploding the canon: Children's literature and the revolution of criticism. En Cano Vela A. y Perez Valverde C. (eds). Canon y literatura infantil y juvenil y otras literaturas: actas del VII congreso internacional de la Sociedad española de didáctica de la lengua y literatura (pp. 21-30). Cuenca: Ediciones de la Universidad de Castilla la Mancha.

Jauregui C., Moraña M. y Mignolo W. (2008) Coloniality at large: Latinamerica and the postcolonial debate (Latinamerica otherwise). Durham: Duke University Press.

McGillis, R. (2000). Voices of the other: children's literature and the postcolonial context. Children's literature and culture, Vol X. Nueva York y Londres: Garland.

Nichols G. (2001). Wha me mudder do en Mac Grough, R. (ed.) 100 Best Poems for Children. Londres: Puffin. 
Nodelman, P. (1992). The other: Orientalism, colonialism, and children's literature, Children's Literature Association Quarterly, 17: 29-35.

Paul, L. (2005). Sex and the children's book en The Lion and the Unicorn, 29 (2): 222-35.

Spivak G. (1988). Can the subaltern speak? En Nelson C. y Grossberg L. (eds) Marxism and the Interpretation of Culture (pp 24-28). Londres: Macmillan.

Reynolds, K. (2007). Radical children's literature: future visions and Aesthetic transformations in juvenile fiction. Basingstoke: Palgrave Macmillan.

Said E. (1996). Cultura e imperialismo. Barcelona: Anagrama

Sánchez Prado, I. (2011). Is the postcolonial South Asian? A Latin American reply. Ponencia en Congreso Modern Language Association, Los Angeles. http://ignaciosanchezprado.blogspot.com/2011/01/is-postcolonial-south-asianlatin.html.

\footnotetext{
${ }^{1}$ Las traducciones de todas las citas son de la autora del presente artículo.

2 El concepto surgió en Canadá en un informe publicado en 1970 por la Royal Comission on Bilingualism and Biculturalism, en el que se concluye que "Canadá es un país oficialmente bilingüe pero fundamentalmente multicultural”.

${ }^{3}$ Según Lisa Paul, "conforme el siglo XX iba tocando a su fin parecía claro que el multiculturalismo no iba a hacer posible que el mundo cantase en perfecta armonía y algunos académicos como Said pusieron su atención en identificar posibles causas de disonancia (...). El trabajo pionero de Said fue de gran influencia pues llamó la atención sobre lo que había sido una implícita asunción sobre el orden mundial normal (colonial)” (2005: 231).

${ }^{4}$ Se puede explicar por la falta de reconocimiento de la corriente dominante en los estudios poscoloniales de realidades poscoloniales distintas de las derivadas del imperialismo británico, como pueden ser las derivadas del antiguo imperio español, a lo que Ignacio M. Sánchez Prado se ha referido recientemente como el "incómodo estatus de América Latina en lo pos-colonial” (2011).

${ }^{5}$ Célebre frase acuñada por Salman Rushdie, introducida por primera vez en una intervención en un programa de televisión y posteriormente utilizada por él mismo como título de un artículo ("The Empire Writes Back with a Vengeance").

${ }^{6}$ El término "subalternidad", utilizado en los estudios poscoloniales, denota la posición de exclusión social y marginalidad en que vive el colonizado.

${ }^{7}$ El concepto doble colonización hace referencia al paralelismo establecido entre el estatus de las mujeres (sometidas a una autoridad patriarcal) y los colonizados (sometidos al colonizador). Según Spivak, "si en el contexto de la producción colonial, el subalterno no tiene historia y no puede hablar, el subalterno femenino está aún más en la sombra” (1998:28).
}

\section{Referencias de la autora}

María Luisa Alonso es licenciada en Ciencias Políticas y Master en didáctica de la lengua y literatura y en libros infantiles y juveniles. Reside en Luxemburgo donde trabaja para una biblioteca infantil intercultural coordinando sus actividades, animando talleres de lectura para niños y promocionando la literatura infantil y juvenil en este país. En la actualidad está preparando una investigación doctoral dirigida a analizar comparativamente textos contemporáneos para jóvenes lectores escritos por grupos poscoloniales residentes en Europa.

Email: marialuisalonso@yahoo.es

Para citar este artículo:

Alonso Soroa, M.L. (2012). Estado de la cuestión sobre la perspectiva poscolonial: Subversión de conceptos en literatura infantil y juvenil actual. Bellaterra Journal of Teaching \& Learning Language \& Literature, 5(1), 7989. 